\title{
The 3D-Structure Analysis of Spermatids in the Seminiferous Epithelia by the Serial Block-Face SEM Method
}

\author{
Yuji Hasebe ${ }^{1}$, Tomohiro Haruta ${ }^{1}$, Natasha Erdman ${ }^{2}$, Mistuo Suga ${ }^{1}$, Hideo Nishioka ${ }^{1}$, Toshiaki \\ Suzuki ${ }^{1}$ \\ 1. JEOL Ltd, 3-1-2 Musashino, Akisima, Tokyo 196-8558 Japan \\ 2. JEOL USA inc, 11 Dearborn Road Peabody, MA 01960 USA
}

In a seminiferous epithelium, spermatogenesis is observed from a spermatogonium to sperm. In the spermatogenesis, cells undergo meiosis and mitosis while the spermatogonium differentiates into the sperm. These cell divisions are not complete and the cells are still connected by intercellular bridges even after cell divisions until sperm formations. In order to understand the network of the cells, it is necessary to analyze more than several tens of the cells, which are connected by the intercellular bridges. Therefore, it is difficult to analyze 3-dimensional structure of the network of the cells by TEM-tomography and confocal laser scanning microscopy (CLSM), because TEM does not have enough analysis area, and CLSM does not have enough spatial resolution. To solve this problem, we applied a serial block-face SEM (SBF-SEM) method $^{1)}$ to analyze the connections of the cells. In the SBF-SEM method, an ultramicrotome is installed in a specimen chamber of a SEM. The surface of a resin embedded specimen is cut at predetermined thickness, and the SEM image of the exposed specimen surface is captured. By repeating this process and stacking the captured SEM images, it is possible to reconstruct the 3-dimensional cell structure in high resolution at nm level for large volume of sub millimeter cubic.

In this study, we used field-emission SEM, JSM-7100F (JEOL Ltd., Japan), equipped with the Gatan 3View 2XP(Gatan Inc., USA) (Figure 1). We took about 1,000 SEM images of the sliced seminiferous epithelium, and reconstructed its 3-dimensional structure. We also extracted the cells connected by the intercellular bridges from the reconstructed structure. The analyzed area of this data is $80 \mu \mathrm{m}$ (X direction), $90 \mu \mathrm{m}$ (Y direction), and $70 \mu \mathrm{m}$ (Z direction) (Figure 2). We recognized about 50 connected spermatids, and found the spermatid with 6 intercellular bridges, meaning number of cell division is 6 for this cell. We also inferred order of cell divisions for these 50 cells from number of the intercellular bridges. We report the details of the analysis in the presentation.

\section{References}

[1] Denk W. and Horstmann H., Serial block-face scanning electron microscopy to reconstruct three-dimensional tissue nanostructure. Plos Biol (2004), 2, P. 1900-1909. 


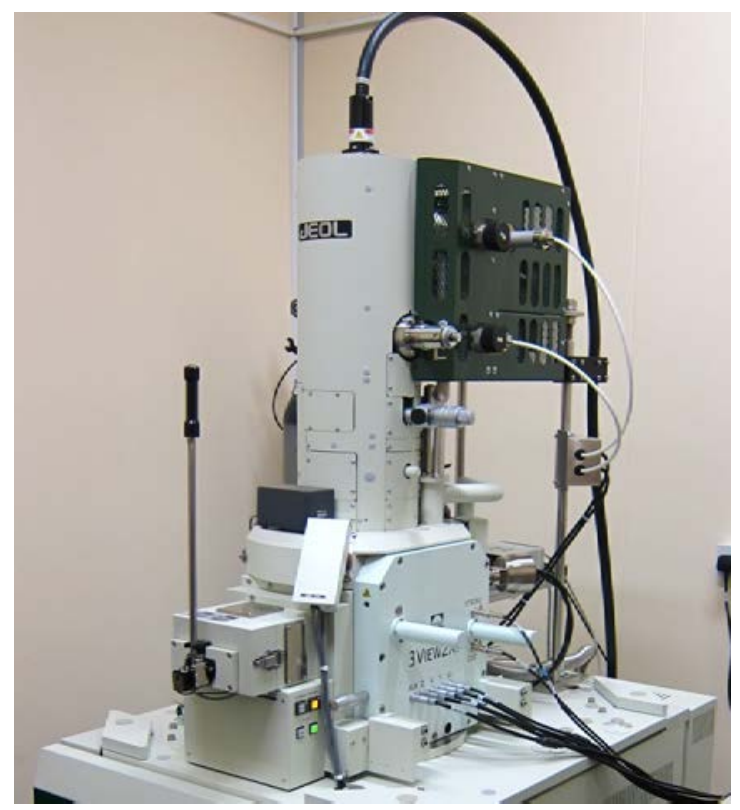

Figure1. This figure shows JSM-7100F, equipped with the Gatan 3View 2XP.

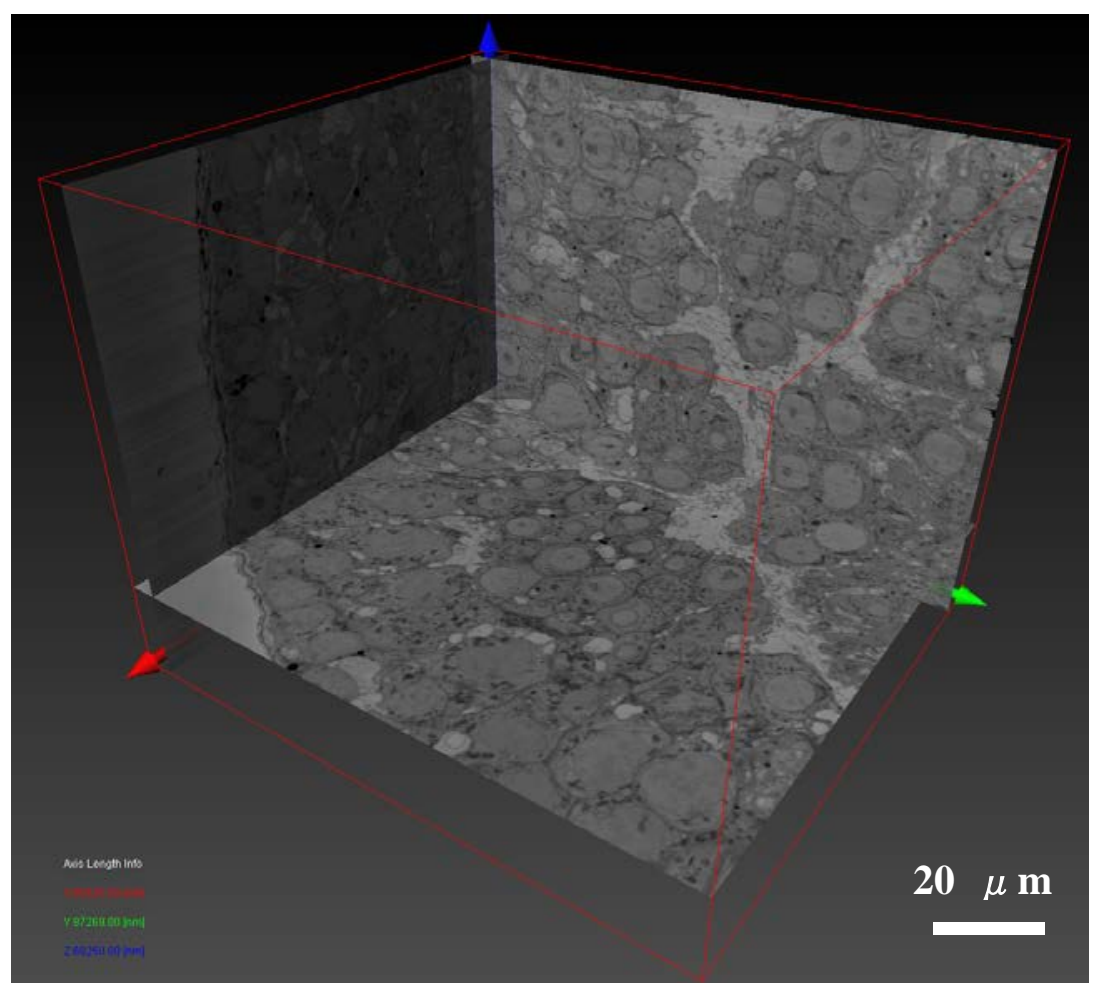

Figure2. This figure shows orthoslices of a seminiferous epithelium. 